\title{
Control Strategy of Energy Storage Buffer System for Charging Station with V2G Function
}

\author{
Shuguang $\mathrm{Liu}^{1}$, Huawei $\mathrm{Xie}^{2}$ and Wenpu Zhao ${ }^{2}$ \\ ${ }^{1}$ School of Mechatronic Eng., Huangshan University, Huangshan 245041, China \\ ${ }^{2}$ School of Electronic and Information Eng., Xi'an Polytechnic University, Xi' an 710048, China
}

\begin{abstract}
Electric vehicle (EV) charging stations based on the V2G (Vehicle to Grid) technology support bi-directional energy interaction between grid and electric vehicles. The energy flow from the grid will be injected into the battery when the battery needs to be charged. While the electric vehicle is in a suspended state, the energy will flow from electric vehicles to grid so as to improving the energy utilization. By using the energy storage buffer system, we can compensate the pulse power of EV's fast charging and reduce the adverse influence on the distribution network caused by the fast charging stations. This paper studies the topology structure of fast charging station with energy storage buffer system and the fast charging power characteristics of different types of batteries. Then taking the distribution network real power change limit into account, we propose an operation mode of the energy storage buffer system of the fast charging stations and its current control strategy. Furthermore, to design the storage buffer system module and its control parameters, we combine the configuration of fast charging stations to deduce the operation parameters of the storage buffer system.
\end{abstract}

Keywords-electric vehicle; charging station; V2G; load fluctuation

\section{INTRODUCTION}

Electric vehicles and power battery for smart grid plays two different roles. On the one hand, electric vehicle power service as a huge load, would be likely to occupy the entire great proportion of the power load, its operation safety for power grid will have a significant impact; on the other hand, as a kind of energy storage device, the power battery of electric vehicle is a kind of active resource to protect and optimize the operation of power grid. Therefore, how to make electric vehicles and their power batteries in these two roles between the smooth and free conversion, is one of the key issues in the combination of smart grid and electric car. This is specifically reflected in how to reasonably arrange the load of electric vehicles and how to optimize the operation of power battery. When the electric vehicle as a load, we can arrange the charging time reasonably through economic means and technical means, to realize the orderly charge management, achieve the effect of shifting peak and filling valley, improve the efficiency of the system and reduce the influence of the grid security. When power battery as energy storage device, it can be used as spare capacity of the system, or the peak load to the power grid to provide electricity, optimizing the power grid operation, improve the safety and reliability of the system. In this context, the concept of V2G (vehicle to grid) arises at the historic moment.
Large-scale electric vehicle charging stations construction will affect the safe operation of the power distribution network, especially in those big power fast charging stations which will bring strong impact to the grid[1-2]. Fast charging technology is the basis of the wide application of electric vehicles, which has great influence on the development of electric vehicles. The charging rate is mainly controlled by two aspects: the best charging rate of the battery and the charging capacity of the charging station[3-4].

In this paper, taking the distribution network real power change limit into account, we propose an operation mode of the energy storage buffer system of the fast charging stations and its current control strategy. Furthermore, to design the storage buffer system module and its control parameters, we combine the configuration of fast charging stations to deduce the operation parameters of the storage buffer system.

\section{EV's FAST CHARGING STATIONS INCLUDING STORAGE BUFFER SYSTEM}

\section{A. EV Fast Charging Power Characteristics}

Electric vehicle charging station contains AC and DC charging pile[5-6]. AC charger charging voltage is $220 \mathrm{~V}$, rated power is $3.5 \sim 7 \mathrm{~kW}$. DC charging pile adopts $400 \mathrm{~V}$ or $750 \mathrm{~V} \mathrm{DC}$ charging voltage, rated power is $50 \sim 175 \mathrm{~kW}$.

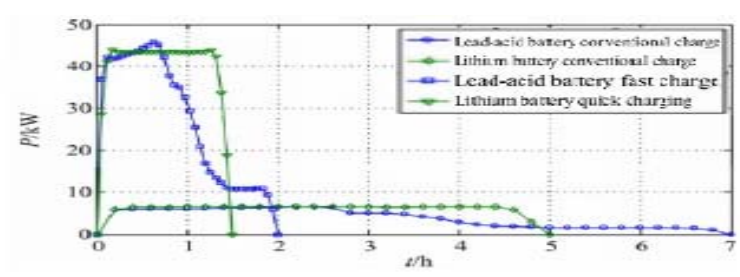

FIGURE I. EV'S CHARGING POWER CHARACTERISTICS

Electric vehicles quick charge, is realized by using an not on-board DC charging mode is shown in Fig I for lead-acid battery and lithium battery electric vehicle use the fast charging and power characteristics of conventional charging ways[7-8]. Electric vehicle charging power characteristics is determined by the battery type and charging ways, in conventional lithium-ion batteries and quick charge approximate constant power, while lead-acid battery charging process under two kinds of charging ways are two stages to recharge. Lead-acid 
battery charging the conventional peak power of $6.50 \mathrm{~kW}$, and using quick charge will reach its peak power is $45.80 \mathrm{~kW}$.

\section{B. EV's Fast Charging Station Structure Including Energy Storage Buffer System}

Fig II is the structure of electric vehicle fast charging station including storage buffer system, charging stations attached to a certain number of DC charging machine. In the fast charging station DC bus increase storage buffer system, in order to smooth the rapid charging load caused by volatility, shorten the distribution network active rate the charging time of the electric vehicle.

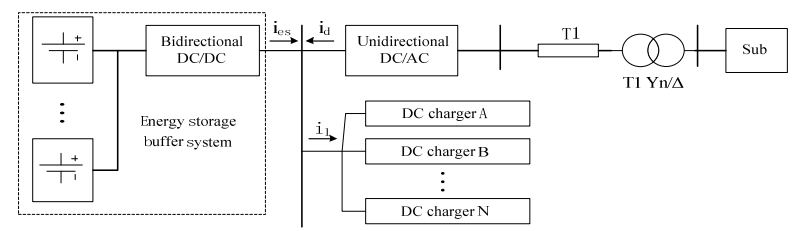

FIGURE II. THE STRUCTURE OF EV'S FAST CHARGING STATION INCLUDING STORAGE BUFFER SYSTEM

\section{StOrage Buffer System's RunNING OF FAST CHARGING STATION}

Fast charging stations in a rapidly changing DC charging machine power, must be active storage buffer system of the compensation gap. Fig III for lead-acid battery fast path approximation that $\mathrm{DC}$ bus voltage constant. When setting $\mathrm{t}=\mathrm{t}_{1}$, $\mathrm{i}_{1}$ order fast charging load leap to ill (Fig.3 (a)), due to the injection current distribution network id set according to slope $\mathrm{R} 1=\mathrm{i}_{12} /\left(\mathrm{t}_{2}-\mathrm{t}_{1}\right)$ gradually increase. At this time can't meet the demand of charging load of power distribution network, requires the power balance charge storage of the compensation. As shown in Fig.3 (b) and (c), energy storage injection current $i_{\text {es }}$ is equal to the load current $i_{1}$ and power injection current differential value of $i_{d}$.

When $\mathrm{t}=\mathrm{t}_{2}$ distribution network can meet the demand of load of power alone, storage buffer system in standby state. When the electric vehicle charging is completed, entering a low power energy storage slow charging status, for the next pulse charging reserve power. Electric vehicle charging load reduced, current distribution network id to set up the slope $\mathrm{R}_{2}$ decreases $\left(\mathrm{R}_{2}>>\mathrm{R}_{1}\right)$. In Fig.3 (b) due to the change of the distribution network current rate limit is greater than the load current decline rate, id meet charging load current changes at this time. When the electric vehicle battery is lithium battery, the fast charging current $i_{1}$ step down, after the completion of distribution network current $i_{d}$ set according to the slope $R_{2}$ decreases, and only will absorb the excess electricity energy storage buffer system.

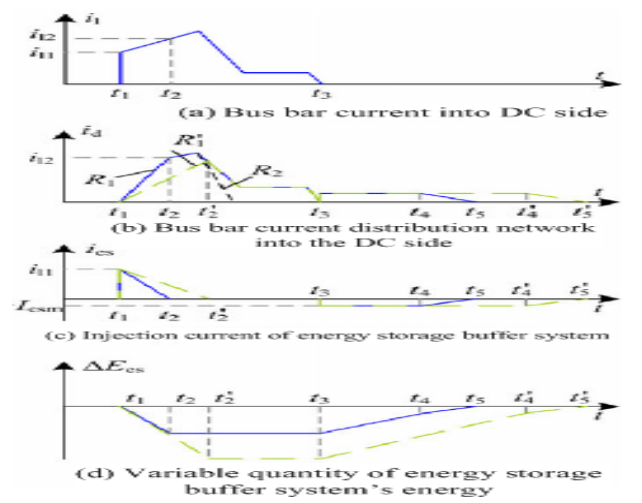

FIGURE III. STORAGE BUFFER SYSTEM OPERATION MODE

When $\mathrm{t}=\mathrm{t}_{3}$ energy storage buffer system to enter low power slow charging status, charge current depends on the size of the energy storage is full of the energy required. At $t=t_{3} \sim t_{4}$ initial stage charging current is constant -Iesm, although at $t=t_{3}$ time charging current step change, but as a result of Iesm small, slow charging flow distribution network can be directly meet the energy storage system. At $t=t_{4} \sim t_{5}$ stage, $i_{\text {es }}$ and energy storage buffer system of energy gaps $\Delta$ Ees is proportional, as shown in Fig.3(d) for energy storage buffer system energy change during charge and discharge. At $\mathrm{t}=\mathrm{t}_{5}$, charge storage buffer system, into the standby state. When the setting of injection current slope distribution network for $\mathrm{R}_{1^{\prime}}\left(\mathrm{R}_{1^{\prime}}<\mathrm{R}_{1}\right), \mathrm{t}=\mathrm{t} \sim \mathrm{t}_{2}$, phase increases energy storage system of injection current, and the release of energy also increases.

\section{Energy Storage BufFer System CONTROL STRATEGY}

Multiphase crisscross parallel non isolated Buck/Boost bi-directional DC converter has the advantages of low power devices and low conduction loss, etc [9], is suitable for charging station storage buffer system of high power DC transform. This paper adopts three-phase alternating parallel Buck/Boost DC converter, circuit structure as shown in Fig IV, which consists of multiple modules and series-parallel storage units transformer high voltage side.

Low voltage side filter inductance $\mathrm{L}_{1} \sim \mathrm{L}_{3}$ and rapid charger DC bus is linked together, using the three-phase inductance current $\mathrm{i}_{\mathrm{L} 1} \sim \mathrm{i}_{\mathrm{L} 3}$ crisscross superimposed DC current ripple can be reduced [9], $\mathrm{CH}$ and $\mathrm{CL}$ of high and low voltage side filter capacitance respectively. To overcome Buck and the Boost model independent control of two freedom limited switch, the deficiency of the single channel power switch Siu and Sid (I=1, $2,3)$ driven by complementary way $[10,11]$, in the same way for two-way current controlled by a single controller.

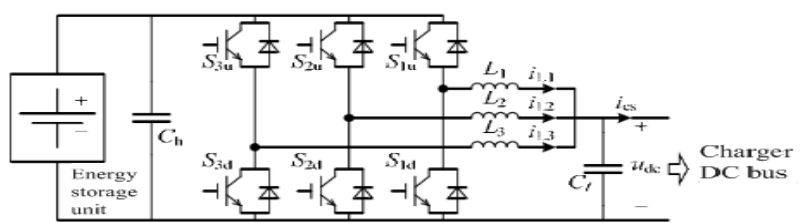

FIGURE IV. STORAGE BUFFER SYSTEM DC CONVERSION CIRCUIT 


\section{A. Control Strategy of Energy Storage Buffer System}

Fig V shows the storage buffer system control structure, using the fast charging load current $i_{1}$ and set distribution injection current increase and decrease of slope $R_{1}, R_{2}$, inject current $i^{*}{ }_{d}$ estimation of distribution network, with energy storage charge current reference $i^{*}$ ch and load current $i_{1}$ get energy storage current reference $i^{*}$ es. DC converter inner ring use the low voltage side inductance current tracking control, by the phase inductance current reference $i_{\mathrm{L1}}^{*} \sim \mathrm{i}_{\mathrm{L} 3}^{*}$ and the actual value of $i_{L 1} \sim i_{L 3}$ duty ratio deviation by PI. Switch trigger link again according to the saw tooth wave signal (phase mutual crisscross Ts $/ 3$, Ts is the saw tooth wave cycle) and duty ratio is presupposed, determine the power switch $\mathrm{S}_{1 \mathrm{u}} \sim \mathrm{S}_{3 \mathrm{u}}$ and complementary $S_{1 d} \sim S_{3 d}$ trigger signal.

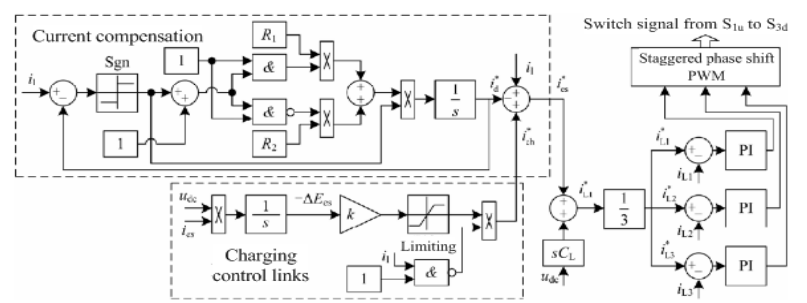

FIGURE V. STORAGE BUFFER SYSTEM CONTROL BLOCK DIAGRAM

When the electric vehicle in the process of quick charge $\mathrm{t}=\mathrm{t}_{1} \sim \mathrm{t}_{3}$, and $\mathrm{i}_{1}>\mathrm{i}_{\mathrm{d}}^{*}$ after logical operation by the integrator output at predetermined slope increased $\mathrm{R}_{1}$, until $\mathrm{i}_{\mathrm{d}}^{*}=\mathrm{i}_{1}$ unchanged; When $\mathrm{i}_{1}<\mathrm{i}_{\mathrm{d}}{ }_{\mathrm{d}}$ integrator output when $\mathrm{i}_{\mathrm{d}}{ }_{\mathrm{d}}$ at a predetermined slope $R_{2}$ decreases, and the injection current $i_{d}{ }_{d}$ estimate distribution network. Charging control links of current $i^{*}{ }_{c h}$ is equal to zero, I will get the energy storage discharge current $i_{1}$ minus the $i^{*}$ reference $i_{\text {es }}^{*}$, quick charge compensation in distribution network into charging power gaps. In the process of the electric vehicle quick charge, if the residual capacity of battery energy storage system is less than the setting threshold, exit run wrong distribution network power storage buffer system gaps to compensate.

Through logic to ensure that the storage buffer system of charging management and energy buffer is not at the same time, after the completion of the electric vehicle charging $i_{1}$ and $i^{*}{ }_{d}$ are 0 , charging control links to the charging current reference value calculated for energy storage. Store the actual current ies and DC bus voltage $V_{d c}$ after integral to get the energy storage system in the process of charging and discharging energy change $\Delta$ Ees. Energy storage charge current reference ies and $\Delta$ Ees is proportional, by limiting link limit between $-\mathrm{I}_{\text {esm }}$ to 0 , in the process of energy storage charging current reference $i_{\text {es }}^{*}$ equals $i^{*}$ ch. The energy storage unit using voltage source ues and internal resistance $R_{e s}$, equivalent to single channel conversion circuit as shown in Fig VI (a). Set the $\mathrm{S}_{\text {iu }}$ duty ratio as $\mathrm{d}$, and increased disturbances on the steady state point that $\mathrm{d}$ $=\mathrm{D}+\Delta \mathrm{d}, \mathrm{i}_{\mathrm{L}}=\mathrm{I}_{\mathrm{L}}+\Delta \mathrm{i}_{\mathrm{L}}, \mathrm{u}_{\mathrm{h}}=\mathrm{U}_{\mathrm{h}}+\Delta \mathrm{u}_{\mathrm{h}}, \mathrm{u}_{\mathrm{dc}}=\mathrm{U}_{\mathrm{dc}}+\Delta \mathrm{u}_{\mathrm{dc}}, \mathrm{u}_{\mathrm{es}}=\mathrm{U}_{\mathrm{es}}+$ $\Delta \mathrm{u}_{\mathrm{es}}, \mathrm{i}_{\mathrm{es}}=\mathrm{I}_{\mathrm{es}}+\Delta \mathrm{i}_{\mathrm{es}}$, According State-Space Averaging Method and ignore the high order component can obtain small signal model of the system

$$
\left[\begin{array}{c}
\Delta \dot{i}_{L} \\
\Delta \dot{i}_{l h} \\
\Delta \dot{i}_{l d c}
\end{array}\right]=\left[\begin{array}{ccc}
\frac{-R_{L}}{L} & \frac{D}{L} & \frac{-1}{L} \\
\frac{-D}{C_{h}} & \frac{-1}{R_{e s} C_{h}} & 0 \\
\frac{1}{C_{I}} & 0 & 0
\end{array}\right]\left[\begin{array}{l}
\Delta i_{L} \\
\Delta u_{h} \\
\Delta u_{d c}
\end{array}\right]+\left[\begin{array}{c}
\frac{U_{h}}{L} \\
\frac{-I_{L}}{C_{h}} \\
0
\end{array}\right] \Delta d+\left[\begin{array}{cc}
0 & 0 \\
\frac{1}{R c s C_{h}} & 0 \\
0 & 0
\end{array}\right]\left[\begin{array}{l}
\Delta u_{e s} \\
\Delta i_{e s}
\end{array}\right]
$$

Let $\Delta u_{e s}=0, \Delta i_{e s}=0$, from the formula (1) we can obtain

$$
\begin{gathered}
\Delta i_{L}=\frac{D}{R_{L}+L_{S}} \Delta u_{h}-\frac{1}{R_{L}+L_{S}} \Delta U_{d c}+\frac{U_{h}}{R_{L}+L_{S}} \Delta d \\
\Delta u_{h}=\frac{-R_{e s} D}{R_{e s} C_{h} S+1} \Delta i_{L}-\frac{R_{e s} I_{L}}{R_{e s} C_{h} S+1} \Delta d \\
\Delta u_{d c}=\frac{1}{C_{1} S} \Delta i_{L}
\end{gathered}
$$

Considering (2), (3) and (4), we can obtain

$$
G_{i d}(s)=\frac{\Delta i_{L}}{\Delta d}=\frac{U_{h} C_{1}\left(R_{e s} C_{h} s+1\right)-D I_{L} R_{e s} C_{1} s}{\left(L C_{1} s^{2}+R_{L} C_{1} s+1\right)\left(R_{e s} C_{h} s+1\right)+D^{2} R_{e s} C_{1} s}
$$

According to the formula (5) and Fig.5 available DC transform current control structure as shown in Fig.6 (b), using energy storage current reference $i^{*}$ es superposition capacitive current $\mathrm{sC}_{\mathrm{L}} \mathrm{U}_{\mathrm{dc}}$ inductor current reference $\mathrm{i}_{\mathrm{L}}$, inductance current deviation signal by the regulator $\mathrm{G}_{\mathrm{c}}(\mathrm{s})$ and the modulation link generated after duty ratio $d$, then by formula (5) get $i_{L}$ about $d$ small signal transfer function. Which use PI regulator $\mathrm{Gc}(\mathrm{s})=$ $\mathrm{kp}+\mathrm{ki} / \mathrm{s}$, for open loop transfer function

$$
G_{i}(s)=\frac{k_{p}+k_{i} / s}{V_{m}} G_{i d}(s)
$$

Type: $k_{p}$ and $k_{i}$ are the proportional and integral coefficient of current compensation control; The $\mathrm{V}_{\mathrm{m}}$ is saw tooth wave amplitude value. According to the DC conversion circuit parameter formula (6) can be choose to PI compensating link parameters.

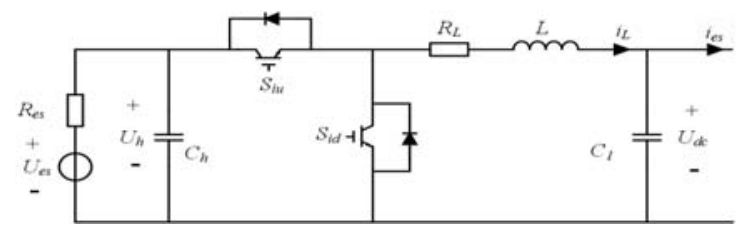

(a) Single channel equivalent circuit 


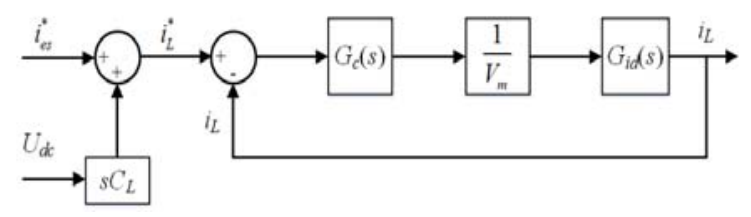

(b) Current control structure

FIGURE VI. SINGLE-CHANNEL DC TRANSFORM CIRCUIT AND CURRENT CONTROL STRUCTURE

\section{B. Estimation of Energy Storage Buffer System's Running Parameters}

For energy storage parameter design of buffer system, it is necessary to calculate energy storage capacity and charge and discharge time. As shown in Fig.3(c), during the discharge energy storage buffer system, energy release of the energy storage element

$$
\Delta E_{e s}\left(t_{2}\right)=\frac{u_{d c}}{2} i_{11}\left(t_{2}-t_{1}\right)=\frac{u_{d c} i_{11} i_{12}}{2 R_{1}}
$$

Among them: $\mathrm{R}_{1}=\mathrm{i}_{12} /\left(\mathrm{t}_{2}-\mathrm{t}_{1}\right)$ to a predetermined current slope distribution network, $\mathrm{i}_{12}$ determined by electric vehicle charging power and $\mathrm{R}_{1} ; \Delta \mathrm{E}_{\mathrm{es}}(\mathrm{t} 2)$ of the energy storage unit in the energy released during the process of discharge, the selected energy storage unit capacity must be greater than or equal to $\Delta \mathrm{E}_{\mathrm{es}}\left(\mathrm{t}_{2}\right)$. Fig.3 (c) in the charging process of energy storage system includes constant current charging and charging current attenuation in two stages, total energy storage charge time $\Delta \mathrm{t}_{\mathrm{ch}}=\mathrm{t}_{4}-\mathrm{t}_{3}+\mathrm{t}_{5}-\mathrm{t}_{4}=\Delta \mathrm{t}_{43}+\Delta \mathrm{t}_{54}$, including $\Delta \mathrm{t}_{43}$ for constant current charging time, $\Delta \mathrm{t}_{54}$ recharge current decay time.

\section{1) Constant current charging stage}

At $\mathrm{t}=\mathrm{t}_{2} \sim \mathrm{t}_{3}$ stage energy storage system is in standby state and $\Delta \mathrm{E}_{\text {es }}\left(\mathrm{t}_{3}\right)=\Delta \mathrm{E}_{\mathrm{es}}\left(\mathrm{t}_{2}\right)$, as shown in Fig.3(d). If for constant current charging $-\Delta \mathrm{E}_{\mathrm{es}}\left(\mathrm{t}_{3}\right)>\mathrm{u}_{\mathrm{dc}} \mathrm{I}_{\mathrm{esm}} \mathrm{T}_{\mathrm{r}}$, in $\mathrm{t}=\mathrm{t}_{3 \sim} \mathrm{t}_{4}$ stage charging current $i_{\text {es }}$ constant $I_{\text {esm }}$; If $-\Delta \mathrm{E}_{\text {es }}\left(t_{3}\right)<u_{d c} I_{\text {esm }} T_{r}$, charging current $\mathrm{i}_{\text {es }}$ is slow attenuation, at this time without the constant current charging stage $\Delta \mathrm{t}_{43}=0$ and $\Delta \mathrm{E}_{\mathrm{es}}\left(\mathrm{t}_{4}\right)=\Delta \mathrm{E}_{\mathrm{es}}\left(\mathrm{t}_{3}\right)$, including the $T_{r}$ is the remainder of the energy storage constant current with time. Adopt the way makes the energy storage energy change when $\mathrm{t}=\mathrm{t}_{4}$ for $\Delta \mathrm{E}_{\mathrm{es}}(\mathrm{t} 4)=-\mathrm{u}_{\mathrm{dc}} \mathrm{I}_{\text {esm }} \mathrm{T}_{\mathrm{r}}$, the constant current charging time can be calculated by the formula (8)

$$
\Delta t_{43}=\left\{\begin{array}{rr}
\frac{-v_{d c} I_{e s m} T_{r}-\Delta E_{e s} t_{3}}{v_{d c} I_{e s m}}=\frac{i_{11} i_{12}}{2 R_{1} I_{e s m}}-T_{r}, & \frac{i_{11} i_{12}}{2 R_{1} I_{e s m}}>T_{r} \\
0 \quad, 0<\frac{i_{11} i_{12}}{2 R_{1} I_{e s m}} \leq T_{r}
\end{array}\right.
$$

\section{2) Charging current attenuation phase}

Energy storage buffer system in the process of charging current slow attenuation, the absolute value of charging current $i_{\mathrm{es}}$ and energy storage energy variation $-\Delta E_{\mathrm{es}}$ is proportional to the $i_{\mathrm{es}}=k\left(-\Delta E_{\mathrm{es}}\right)$. Charging control links in Fig.5, $k=-1 /\left(u_{\mathrm{dc}} T_{\mathrm{r}}\right)$, attenuation charging current is satisfied

$$
\begin{aligned}
i_{e s}(t) & =\frac{\Delta E_{e s}(t)}{u_{d c} T_{r}}=\frac{\Delta E_{e s}\left(t_{4}\right)}{u_{d c} T_{r}}-\frac{1}{T_{r}} \int_{t_{4}}^{t} i_{e s}(t) d t \\
& =-I_{e s m}-\frac{1}{T_{r}} \int_{t_{4}}^{t} i_{e s}(t) d t
\end{aligned}
$$

Please type linear differential equation (9) attenuation charging current is available

$$
i_{e s}(t)=\left\{\begin{array}{l}
-I_{e s m} e^{\frac{t_{4}-t}{T_{r}}}, \quad \frac{i_{11} i_{12}}{2 R_{1} I_{e s m}}>T_{r} \\
\frac{i_{11} i_{12}}{2 R_{1} T_{r}} e^{\frac{t_{4}-t}{T_{r}}}, 0<\frac{i_{11} i_{12}}{2 R_{1} I_{e s m}} \leq T
\end{array}\right.
$$

If $\mathrm{i}_{\mathrm{es}}(\mathrm{t})=0.05$ Iesm storage buffer system when charging is completed, by formula (10) available current attenuation charging time, such as formula (11), add those formula (8) and formula (11) can get the total charging time of storage buffer system $\Delta \mathrm{t}_{\mathrm{ch}}$.

$$
\Delta t_{54}=\left\{\begin{array}{l}
3 T_{r}, \frac{i_{11} i_{12}}{2 R_{1} I_{e s m}}>T_{r} \\
\left(\ln \frac{10 i_{11} i_{12}}{R_{1} I_{e s m}}\right) T_{r}, 0<\frac{i_{11} i_{12}}{2 R_{1} I_{e s m}} \leq T_{r}
\end{array}\right.
$$

\section{CONCLUSION}

Construction is booming and development of smart grid, and at the same time, the large-scale promotion and running of the electric vehicle rather than by chance. How will the electric vehicle services and operations and set up and operation of smart grid and perfectly combined, to realize the seamless connection, the integration is a key issue. How to avoid V2G inherent obstacle and perfect play the advantages of power battery for power grid operation, become the electric car and the key to smart grid technology.

Electric vehicles fast charging pulse power charge will be harmful to distribution network, according to the distribution network dispatching demands for fast charging power rate limit, put forward the charging stations in the storage buffer system operation mode and control strategy. The proposed control scheme to make fast charging stations have certain power regulation ability, is advantageous to the electric vehicle charging station further realize the unidirectional or bidirectional Vehicle to grid (Vehicle to grid) technology.

\section{ACKNOWLEDGEMENT}

This work is supported by National Nature Science 
Foundation under Grant 51177115.

\section{REFERENCES}

[1] ZHAO Jun-hua, WEN Fu-shuan, YANG Ai-min, et al. Impacts of electric vehicles on power systems as well as the associated dispatching and control problem. Automation of Electric Power Systems, Vol.35, No.14, pp.2-10, 29, 2011

[2] XU Guo-jun, LIU Yong-sheng, LI Ti-yin, et al. Study on the impact of electric vehicles charging load integrated into distribution network based on analytic hierarchy process and probabilistic simulation. Power System Protection and Control, Vol.40, No.22, pp.38-45, 2012.

[3] Etezadi A M, Choma K, Stefani J. Rapid charge electric vehicle stations. IEEE Trans. on Power Delivery, Vol.25, No.3, pp.1883-1887, 2010.

[4] Bae S, Kwasinski A. Spatial and temporal model of electric vehicle charging demand. IEEE Trans. on Smart Grid, Vol.3, No.1, pp.394-403, 2012 .

[5] QC/T841-2010 Conductive charging interface for electric vehicle[S].

[6] ZHOU Feng-quan, LIAN Zhan-wei, WANG Xiao-lei, et al. Discussion on operation mode to the electric vehicle charging station. Power System Protection and Control, Vol.38, No.21, pp.63-66, 71, 2010.

[7] Qian K J, Zhou C K, Allan M, et al. Modeling of load demand due to EV battery charging in distribution systems. IEEE Trans. on Power Systems, Vol.26, No.2, pp.802-810, 2011.

[8] Mendoza A, Argueta J. Performance characterization GM EV1 with Panasonic lead acid battery. California: Southern California EDISON, 2000.

[9] DU Xue-long, LIU Zhi-zhen, WANG Jian, et al. Comparison and analysis on harmonic suppression of EV charging station. Power System Protection and Control, Vol.40, No.19, pp.139-143, 149, 2012.

[10] Zhang J H, Lai J S, Kim R Y, et al. High-power density design of a soft-switching high-power bidirectional dc-dc converter. IEEE Trans. on Power Electronics, Vol.22, No.4, pp.1145-1153, 2007.

[11] ZHANG Guo-ju, TANG Xi-sheng, ZHOU Long, et al. Research on complementary PWM controlled buck/boost bi-directional converter in super capacitor energy storage. Proceedings of the CSEE, Vol.31, No.6, pp.15-21, 2011. 